\title{
GEOGRAFIE GIULIANE DEL MOVIMENTO FUTURISTA
}

\author{
Eliana Moscarda Mirković \\ Università Juraj Dobrila di Pola \\ Dipartimento di studi in lingua italiana
}

\section{Riassunto}

Il Futurismo di Filippo Tommaso Marinetti, indubbiamente uno tra i più impetuosi movimenti letterari italiani del ventesimo secolo, ha voluto raccogliere le fila di un vasto movimento della modernità che attendeva a porre le basi dell'arte del nuovo secolo, imprimendo ad essa un moto sempre più veloce e cadenze di sempre più marcato distacco dal passato.

Nel nuovo clima di restaurazione della letteratura italiana degli anni Venti, alcune realtà periferiche, tra le quali Trieste, hanno risentito di un avanguardismo tardivo, e una parte della produzione letteraria giuliana vivrà la propria stagione tardo futurista.

Il Futurismo giuliano si presenta articolato soprattutto per le premesse date dalla "cultura di frontiera" che contraddistingue la regione, con i suoi caratteri mitteleuropei e la compresenza della componente antropologico-culturale italiana e slovena.

Parole chiave: Futurismo, Italia, movimento, Venezia-Giulia

Il Futurismo è stato, a livello europeo, il primo movimento d'avanguardia organizzato $^{[1]}$. Marcato dalle stimmate di Filippo Tommaso Marinetti, resta indubbiamente tuttoggi uno tra i più rumorosi, aggressivi ed impetuosi movimenti letterari italiani del ventesimo secolo ${ }^{[2]}$.

Sebbene si possa affermare che la ventata di modernità e apertura internazionale portata dal movimento futurista, contrasti con il grigiore provinciale dei crepuscolari ${ }^{[3]}$, entrambe le esperienze sottolineano i tentativi messi in atto dagli intellettuali italiani ( $\mathrm{e}$ primariamente dagli scrittori) per sfuggire al disagio della condizione italiana del primo Novecento $^{[4]}$. Le due esperienze quindi, almeno sotto questo aspetto, possono venir considerate, in prospettiva storiografica, complementari.

Come quello crepuscolare, lo snodo futurista è decisivo per la trasformazione che le due guerre d'inizio del secolo scorso provocheranno nella posizione del letterato verso la cultura nazionale.

La nuova posizione è il risultato di una duplice revisione: da un lato dei registri

[1] Armellini, G., Colombo, A., Letteratura. Letterature. Antologia Primo Novecento, Vol. G, Zanichelli, Bologna, 2005 , p. 165.

[2] Ricov, J., Talijanski futurizam s predcima i potomstvom, Hrvatsko književno društvo sv. Jeronima, Zagabria-Zagreb, 2004 , p. 16.

[3] Armellini, G., Colombo, A., Guida alla letteratura italiana, Zanichelli, Bologna, 1999 (5), p. 483.

[4] Viola, G. E., Gli anni del Futurismo. La poesia italiana nelletà delle avanguardie, Studium, Roma, 1990, p. 33. 
espressivi e tematici, dall'altro degli orizzonti culturali e di riferimento. Il movimento futurista non andrà quindi circoscritto esclusivamente all'ambito letterario, ma proporrà un programma di profondo rinnovamento per tutte le arti e persino un nuovo senso del vivere, nonché nuovi modelli di comportamento ${ }^{[5]}$.

Il nuovo gusto letterario e culturale ha anchesso (come i crepuscolari) un'impronta antidannunziana, ma è più un sentimento contro il dannunzianesimo (inteso quale lutulento oziare in atmosfere di broccati e ricche di asfodeli) che contro D’Annunzio e la sua poesia. Infatti, ne riprende ed esaspera la tensione vitalistico-eroica, l'esaltazione dell'energia irrazionale e della vitalità immediata e aggressiva.

Con la fondazione del movimento, Marinetti sferra un potentissimo attacco alla teoria artistica a lui contemporanea che si esprime in quel paradosso responsabile che è il rifiuto dell'eroe/vate tramite un atto anch'esso eroico e parimenti innovativo ${ }^{[6]}$. Ci si riferisce soprattutto all'estremismo tecnico del linguaggio ed il suo uso in chiave modernizzante. Un linguaggio nuovo, scientificamente pensato per il nuovo che agisce solo in funzione dello scopo prefissato e non soltanto per l'espressione artistica in se stessa.

Ma il Futurismo è anzitutto la voglia di raccogliere le fila di un vasto movimento della modernità che, sotto varie etichette e con varie cadenze in Italia e soprattutto fuori dall'Italia (importantissimo è il contesto francese alle sue origini e il vanto di Marinetti di poter essere protagonista a Parigi ${ }^{[7]}$ ), attendeva "a porre le basi dell'arte del nuovo secolo imprimendo ad essa moto sempre più veloce e cadenze di sempre più marcato distacco dall'antico modo" ${ }^{[8]}$. Il Futurismo raccolse ed in un certo modo "codificò" questa realtà.

Noi vogliamo cantare l'amor del pericolo, l'abitudine all'energia e alla temerità.

Il coraggio, l'audacia, la ribellione, saranno le menti essenziali della nostra poesia.

La letteratura esaltò fino ad oggi l’immobilità pensosa, l'estasi e il sonno. Noi vogliamo esaltare il movimento aggressivo, l'insonnia febbrile, il passo di corsa, il salto mortale, lo schiaffo ed il pugno ${ }^{[9]}$.

Nessun precedente movimento artistico-letterario era stato nella storia della cultura italiana così esplicito e così fecondo di dichiarazioni di poetica e di manifesti programmatici ${ }^{[10]}$.

I manifesti futuristi riguardanti i vari settori artistici saranno almeno una decina; il Futurismo nasce però ufficialmente con il Manifesto del Futurismo, che appare su "Le Figaro" di Parigi il 20 febbraio 1909.

[5] Guglielmino, S., Guida al Novecento. Profilo letterario e antologia, Principato, Milano, 1990 (4), p. I/90.

[6] Cfr. Fantasia, R., Tallini, G., Poesia e rivoluzione. Simbolismo, Crepuscolarismo, Futurismo, Franco Angeli, Milano, 2004 , p. 184.

[7] Così Rojić, S., Lombra del Futurismo nei Balcani d’oggi, in Shades of Futurism. Futurismo in ombra, Atti del Convegno internazionale Princeton 9-10 ottobre 2009, (a cura di) Frassica P., Interlinea, Novara, 2011, p. 168.

[8] Viola, G. E., Gli anni del Futurismo. La poesia italiana nelletà delle avanguardie, cit., p. 38.

[9] Marinetti F. T., Manifesto del Futurismo, in AA.VV., I manifesti del Futurismo, Edizioni Lacerba, Firenze, 1914, p. 6.

[10] Compaiono, a titolo esemplificativo: Manifesto del futurismo, (Pubblicato da "Le Figaro" il 20 febbraio 1909), Marinetti; Uccidiamo il Chiaro di luna, (aprile 1909), Marinetti; Manifesto dei Pittori futuristi, (11 febbraio 1910), Boccioni, Carrà, Russolo, Balla e Severini; La pittura futurista - Manifesto tecnico, (11 aprile 1910), Boccioni, Carrà, Russolo, Balla e Severini; Contro Venezia passatista, (27 aprile 1910), Marinetti, Boccioni, Carrà, Russolo; Manifesto dei drammaturghi futuristi, (11 gennaio 1911), Marinetti; Manifesto dei Musicisti futuristi, (11 gennaio 1911), Pratella; La musica futurista-Manifesto tecnico, 29 marzo 1911), Pratella; Manifesto della Donna futurista, (25 marzo 1912), Valentine de Saint-Point; Manifesto della Scultura futurista, (11 aprile 1912), Boccioni; Manifesto tecnico della letteratura futurista, (11 maggio 1912), Marinetti; L'arte dei Rumori, (11 marzo 1913), Russolo; Distruzione della sintassi. L'immaginazione senza fili e le Parole in libertà, (11 maggio 1913), Marinetti; L’Antitradizione futurista, (29 giugno 1913), Guillaume Apollinaire; La pittura dei suoni, rumori e odori, (11 agosto 1913), Carrà; Il Teatro di Varietà, (1 ottobre 1913), Marinetti; Il controdolore, (29 dicembre 1913), Palazzeschi; Pittura e scultura futuriste, (1914), Boccioni; Manifesto dell'Architettura futurista, (1914), Sant'Elia; Il teatro futurista sintetico, (1915), Corra, Settimelli, Marinetti; La ricostruzione futurista dell'universo, (1915), Balla, Depero; La Scenografia futurista, (1915), Prampolini; Manifesto del cinema futurista, (1916), Marinetti, Corra, Settimelli; Manifesto dell'Aeropittura futurista, (1929); Manifesto della cucina futurista, (1931), Marinetti; Manifesto futurista della Ceramica e Aereoceramica (1938), Filippo Tommaso Marinetti e Tullio d'Albisola. 
Nel 1915 avverrà il distacco da "Lacerba" ${ }^{[11]}$ e ad esso farà seguito uno spostamento di interessi verso aspetti fino ad allora meno praticati come il teatro, il cinema, la danza.

Il "periodo eroico" del Futurismo durerà fino al maggio del 1920, non senza sussulti interni.

Volontarismo, estremismo, consapevole paradossalità delle affermazioni più recise e iconoclastiche caratterizzano il Futurismo nel periodo eroico: un’operazione che Marinetti portò avanti con abnegazione e il coraggio disinteressati di un vero "missionario dell'arte". ${ }^{[12]}$.

Tra il 1919 e il 1920 si snoda un movimentato dialogo con il fascismo, che culmina nell'uscita di Marinetti dal Fascio il 29 maggio 1920. Il ricongiungimento avverrà intorno al 1923, ma per il Futurismo è iniziato un tempo diverso, con la fase meno fervida del movimento ${ }^{[13]}$.

A partire dal 1920, infatti, il Futurismo appare come un fenomeno ormai archiviato. Nel cuore dell'Italia il gusto di azzardare, sperimentare, gridare e provocare viene gradualmente abbandonato in nome della forma; molti degli scrittori che prima della grande guerra avevano proclamato la necessità della ribellione e del tumulto, dellazione e della dismisura esistenziale e stilistica, ora pronunciano la nuova parola d'ordine di un ritorno all'equilibrio e alla regola.

La rivista che meglio incarna lo spirito nuovo del dopoguerra, legandosi alla lezione prebellica della "Voce" diretta da Giuseppe De Robertis, è «La Ronda». Fondata a Roma nel 1919, avrà vita fino al 1923. Ne fanno parte scrittori di diversa natura e indirizzo, ma accomunati da un doppio rifiuto: il rifiuto delle avanguardie e delle sperimentazioni e il rifiuto di ogni confusione tra letteratura e vita, in virtù del recupero della tradizione letteraria ${ }^{[14]}$.

Sia le pagine dei vociani, sia le pagine dei rondisti si rifaranno a misure frammentistiche, ma con uno spartiacque epocale e di mentalità: mentre i primi hanno insistito sul non-finito e sullo sperimentalismo dello stile spesso incandescente di modi, di toni, di forme; i secondi lavoreranno di fino e di finito, cesellando e levigando fino a togliere ogni impurità, fino ad eliminare qualsiasi disturbo ritmico e stilistico.

Ma intanto nuovi movimenti d'avanguardia, che in parte si rapporteranno al Futurismo, cominceranno a delinearsi in Francia e, in genere, in Europa, tra i quali il Dadaismo e il Surrealismo.

Nel nuovo clima di restaurazione neoclassica della letteratura italiana degli anni Venti, alcune realtà periferiche, tra le quali Trieste, hanno risentito di un avanguardismo tardivo, e una parte della produzione letteraria giuliana vivrà la propria stagione tardo futurista.

Il Futurismo giuliano si presenta articolato soprattutto per le premesse date dalla

[11] "Lacerba" nasce il 1 gennaio 1913 per iniziativa di Giovanni Papini e Ardengo Soffici. Papini e Soffici ritengono che "La Voce" sia troppo impegnata sul piano civile e fondano una rivista artistico-letteraria lontana dal "civismo", dal "filosofume", dal "catonismo", in cui affermano che la sensibilità artistica debba prevalere su ogni altra (Gibellini P., Oliva G., Tesio G., Lo spazio letterario. Storia e geografia della letteratura italiana, Brescia, La Scuola, 1990, p. 753).

[12] De Maria, L., Dondi, L., (a cura di), Marinetti e i Futuristi, Garzanti, Milano, 1994, pp. VII-XLII.

[13] Non vi è dubbio che il Futurismo, almeno sino alla conclusione del Primo conflitto mondiale, sia stato uno dei più originali e prolifici elementi dei movimenti letterari moderni. Un aspetto rimane però oggetto di opposte interpretazioni: la politica futurista ed i suoi rapporti con il fascismo. Per l'argomento si rimanda a Gentile E., Political Futurism and the Myth of the Italian Revolution, in "European cultures. Studies in literature and the Arts" International Futurism in arts and literature, Berghaus G. (a cura di), de Gruyter, Berlin-New York, 2000.

[14] Gibellini, P., Oliva, G., Tesio, G., Lo spazio letterario. Storia e geografia della letteratura italiana, Brescia, cit., p. 805. 
"cultura di frontiera" che contraddistingue la regione, con i suoi caratteri mitteleuropei e la compresenza della componente antropologico-culturale italiana e slovena.

Hace Citra afferma che la Venezia Giulia non abbia sperimentato il primo Futurismo (1908-1914), ma il secondo Futurismo, a cui prenderà parte attivamente, grossomodo, dalla fine della prima fino alla fine della seconda guerra mondiale ${ }^{[15]}$.

In questi anni a Trieste, Gorizia e Udine il futurismo risorgerà a mo' di mille vene carsiche che si dirameranno in un'infinità di iniziative editoriali, teatrali, musicali, jazzistiche e pittoriche ${ }^{[16]}$.

Come precisa Passamani ${ }^{[17]}$, il rapporto della Venezia Giulia col Futurismo scorre su due tempi distinti: il primo (1908-1914) è caratterizzato dall'assenza di un'iniziativa futurista indigena e dall'azione di Marinetti finalizzata soprattutto all'obiettivo nazionalista-interventista, a cui l'istanza estetica è più subordinata che collegata; il secondo (dal 1919) è caratterizzato da artisti i quali, pur operando con più specifiche connotazioni estetiche, non solo entrano in dialettica con le premesse politiche dell'anteguerra, ma anche reagiscono in modi diversi agli avvenimenti nazionali, alle tensioni locali, alle scelte del futurismo postbellico e dell'avanguardia italiana ed internazionale.

La realtà futurista locale si manifesta a partire dal 1919 in coincidenza col riproporsi nel resto d'Italia dell'iniziativa del movimento, impegnato a superare la crisi determinata dalla scomparsa del primitivo nucleo teorico e propulsore. Lidentità del Futurismo, in un primo momento, passa come modello polimorfico e flessibile in un panorama ricco di articolazioni dialettiche, ma anche ideologiche e politiche.

Ma un vero radicamento futurista in ambiente giuliano comincerà a verificarsi solo in seguito, non a Trieste, ma a Gorizia ${ }^{[18]}$.

L'atto di nascita vero e proprio del Futurismo letterario giuliano, va a coincidere con il lancio, appunto a Gorizia, della "Sezione del Movimento Futurista per la Venezia Giulia" ${ }^{19]}$ diretto da Sofronio Pocarini ${ }^{[20]}$ e Mario Vucetich.Prima sono registrabili solo

[15] Hace Citra, R., Carolus L. Cergoly Serini dietro le quinte della pagina, Istituto Italiano di Cultura - Zagreb, EDIT, Fiume-Rijeka, 1996, p. 9.

[16] Salaris, C., Storia del futurismo, Editori riuniti, Roma,1985, pp. 164-167.

[17] Passamani, B., Dall'alcova d'acciaio al Tank ai Macchi 202. Energie futuriste e costruttiviste tra rivolta, utopia e realtà alla frontiera giulia, in Frontiere d'avanguardia. Gli anni del Futurismo nella Venezia Giulia, Catalogo della mostra a cura di Bruno Passamani e Umberto Carpi, Musei Provinciali, Gorizia, febbraio-aprile 1985, p. 18.

[18] Inizierà a manifestarsi "sullo sfondo nazionale della proliferazione organizzativa del Partito Politico Futurista e nel contesto locale del contiguo dramma fiumano; in termini politici, un episodio del postbellico ribollire sovversivo senza radici nella fase eroica dell'interventismo parolibero: una scansione che bisognerà sempre tener presente" Cfr. Carpi, U., Personaggi e vicende della letteratura giuliana d'avanguardia negli anni Venti, in Frontiere d'avanguardia. Gli anni del Futurismo nella Venezia Giulia, Catalogo della mostra a cura di Bruno Passamani e Umberto Carpi, Musei Provinciali, Gorizia, febbraio-aprile 1985, p. 62.

[19] „Movimento futurista. Si è costituita nella nostra città la sezione per la Regione Giulia del Movimento futurista. La dirigono l’architetto Vucetich e il Pocarini. Il programma che verrà svolto è pieno di audacia e di vigore giovanile. Per adesioni: Vucetich, Via Casale 1. La direzione ha emanato il seguente manifesto: Noi fondiamo a Gorizia la Sezione del Movimento Futurista per la Venezia Giulia perché vogliamo: Diffondere nella nostra regione con ogni mezzo tutte le manifestazioni e le affermazioni del Futurismo che distruggono i pregiudizi, le convenzioni che ci legano al vile passato tanto in arte come nella vita pratica e i principi passatisti di morale cretina e pretina che avviliscono e rendono schiavi di falsi pudori.

Sopprimere tutto ciò che puzza di vecchio, di falso, tutti i gesuitismi politici ed artistici e le ipocrisie del sentimentalismo e del romanticume.

Far risorgere le energie giovani frementi di novità.

Vivere e far vivere la nostra vera vita moderna dinamicamente italiana.

Amare l'Italia sopra ogni altra cosa.

Agire futuristicamente da italiani il bene dell'Italia.

Combattere unicamente per l'italianità di tutto ciò che è italiano.

Mario Vucetich architetto e pittore futurista

Sofronio Pocarini poeta e pittore futurista." in "La Voce dell'Isonzo" 11/10/1919.

[20] Emilio Furlani, il giovane goriziano forse più vicino a Pocarini, ricordava fin dal 1924, presentando su "Originalità" la figura 
spunti aneddotici, come il discorso di Marinetti del 20 febbraio 1909 su Trieste la nostra bella polveriera e la serata del 12 gennaio 1910 animata da Mazza, Palazzeschi e Marinetti stesso $^{[21]}$. Entrambi gli avvenimenti ebbero luogo al Politeama Rossetti, sebbene di carattere diverso: strettamente politico il primo, letterario il secondo.

In questa fase iniziale, la cultura triestina esprime nei confronti del futurismo soprattutto ostilità e dissenso e nella provincia giuliana, il formarsi di nuclei d'avanguardia costituisce, prima di trasformarsi in fatto ideologico e in vicenda artistica, un fenomeno generazionale e sociale.

Nel 1923, dattiloscritti in proprio dagli estensori e confezionati in soli dieci esemplari, escono i due fascicoli (febbraio-maggio e giugno-luglio) di "Epeo", un tentativo di laboratorio d'avanguardia e di libreria-editrice, che esibiva una grafica ornamentale di notevole fattura tutta firmata da Giorgio Riccardo Carmelich. Ma vi appaiono anche i nomi di Dolfi e Jablowsky.

Benché Carmelich in "Epeo" evochi il Futurismo come milizia avanguardistica, sarebbe un errore catalogarlo tra i futuristi di stretta osservanza. Troppo triestino e mitteleuropeo Carmelich, per appiattirsi sull'avanguardismo italiano predicato in quello stesso periodo dai marinettiani ortodossi ${ }^{[22]}$.

A Trieste si sta intanto costituendo anche un altro nucleo, questo dichiaratamente futurista, raccolto attorno a Umberto Martelli e a Bruno Giordano Sanzin ${ }^{[23]}$, che diedero vita a un "Gruppo Futurista Studentesco":

Con l'approvazione di Marinetti, direttore del movimento futurista, si è formato a Trieste il "Gruppo Futurista Studentesco": non una congrega di tesserati, ma un gruppo di giovani desiderosi di diffondere l'idee futuriste. Il Futurismo, avanguardia della sensibilità artistica italiana, è sempre in anticipo sulla lenta sensibilità delle masse; rimane perciò spesso incompreso dalla maggioranza, che non può intendere le sue scoperte stupefacenti. Tutti i simpatizzanti desiderosi di prender parte alla vita futurista, sono invitati a mandare la loro adesione in sede ${ }^{[24]}$.

dell'amico, che nella primavera del 1919 (proprio in concomitanza con l'adesione al Partito Futurista) il giovane Sofronio aveva diretto un gruppo "Yoga", "schiera di irruenti e arditi giovani futuristi goriziani" che, come suggerisce il titolo di tale raggruppamento, più che ai programmi futuristi si richiamano allo spiritualismo irrazionale nutrito di teorie esoteriche ed orientaleggianti. "Yoga" aggregò gli elementi più inclini alla trasgressione comportamentale e alla sovversione politica, fra i quali spiccava un manipolo di futuristi capitanato da Cesare e Mino Somenzi. Sul piano artistico non fu certo la tendenza marinettiana a caratterizzare il gruppo, nel complesso nient'affatto macchinolatrico e dinamistico, orientato piuttosto a recepire, attraverso Comisso, gli echi della poetica dei metafisici, da Savinio a De Pisis allo stesso De Chirico.

Pocarini non fu né un poeta né un pittore di grande rilievo, ma fu indubbiamente un animatore eclettico e infaticabile. Pocarini aveva soggiornato, per qualche tempo, come corrispondente a Fiume e in quelloccasione era stato in relazione con la "Testa di Ferro", appunto il richiamo al movimento avanguardistico-libertario "Yoga", lanciato da Guido Keller. Una conferma ulteriore della consuetudine di Pocarini con l'ambiente e il gusto di Keller viene offerto dalla notizia della fondazione da parte sua in Istria (a Portorose) d'una "sezione degli aviatori futuristi con a capo i battaglieri Bonifacio e Boccalatte". Indizi che consentono di affermare, come sostiene Carpi, che la ventata fiumana fu determinante per stimolare ed aggregare il movimento avanguardistico giuliano ai suoi esordi (Carpi U., Futuristi, metafisici e 'spiriti liberi' nella Fiume di D’Annunzio: la "Unione Yoga" di Guido Keller, in "Studi Novecenteschi”, VIII, Pisa, n. 22 dicembre 1981, pp. 133-161). Una molla, quella fiumana, se non eversiva certo inquieta e insofferente, che, nel primo dopoguerra, fa scattare il movimento goriziano e triestino.

Ma nella primavera del 1919 definirsi futurista e yoga significava, innanzitutto, da parte di giovani goriziani esordienti, proclamare delle insofferenze e un tumultuoso bisogno di orientamento e di organizzazione. Cfr. Carpi, U., Personaggi e vicende della letteratura giuliana d'avanguardia negli anni Venti, in Frontiere d'avanguardia. Gli anni del Futurismo nella Venezia Giulia, cit., pp. 62-63.

[21] Per la ricostruzione marinettiana di questi episodi si veda Marinetti F. T., Teoria e invenzione futurista, (a cura di) De Maria, L., Mondadori, Milano, 1968, pp. 210-216.

[22] Carpi, U., 1923-1925: Giornali dell'avanguardismo giuliano, in, Intellettuali di frontiera. Triestini a Firenze (1900-1950), Atti del Convegno (18-20 marzo 1983), Vol. I, a cura di Pertici, R., Olschki, Firenze, 1985, p. 97.

[23] Per i rapporti di Bruno Giordano Sanzin col futurismo si rinvia a Sanzin, B. G., Tappe autobiografiche essenziali, in "ES”, n. 3, febbraio-maggio, 1975, pp. 59-71.

[24] „Gaudeamus Igitur“, n. 2, Trieste, 9 dicembre 1922, p. 2. 
Il gruppo di Sanzin e di Martelli, come nota Carpi ${ }^{[25]}$, tendeva a presentarsi con un crisma di ufficialità e di ortodossia. Emetteva comunicati sul tesseramento, predisponeva campagne di diffusione stampa (in particolare a favore del bollettino ufficiale di Marinetti "Il Futurismo" e, quel che più interessa, del "Der Futurismus" diretto da Ruggero Vasari a Berlino in ambiente sturmista nonché dello stesso "Der Sturm"), svolgeva opera di proselitismo lanciando manifesti divulgativi fatti diligentemente avallare dallo stesso Marinetti e mandava in giro "circolari".

Il tono assunto da questo gruppo futurista risulta pertanto affatto diverso da quello degli avanguardisti di "Epeo": reclute, i primi, della truppa marinettiana; attratti, i secondi, dalla libera sperimentazione. Ed anche a livello ideologico le differenze sono nette, inclinazione europea, piena disponibilità ad accogliere modelli tedeschi ed assenza di punte postcombattentistiche per "Epeo"; connotazioni inequivocabili nel seguente "comunicato" per il secondo gruppo goliardo-futurista: "Ringraziamo tutte quelle gentili signorine, che vollero dimostrare il loro sconfinato amore per i futuristi, dichiarandoli i veri discendenti dell'aggressiva Italia di Vittorio Veneto" ${ }^{[26]}$.

Cogliamo già in nuce, insomma, le ragioni autentiche della reciproca diffidenza, della collaborazione breve e fredda, del rapido ritrovarsi, dopo un tratto di percorso comune, su prospettive divergenti: sempre militante futurista Sanzin, sempre inquieto sperimentatore Carmelich. [...] Il futurismo esercitava naturale attrattiva, col suo prestigio di unico "ismo" italiano, sulle inclinazioni avanguardistiche degli epeiani, ed era altrettanto ovvio che un'ulteriore spinta all'incontro venisse dalla necessita di costituire una sorta di fronte unito contro il comune nemico, contro l'opinione pubblica passatista ${ }^{[27]}$.

Le premesse per una collaborazione futurista dei gruppi d'avanguardia triestini dunque esistevano, malgrado i palesi elementi di disomogeneità. Ne scaturisce una situazione contraddittoria, di cui il frutto più cospicuo di tale collaborazione saranno le "Energie Futuriste", con una direzione non concordata, bensì contesa fra Sanzin e Carmelich.

Un clima analogo si va creando nell'ambito complessivo della Venezia Giulia, dove operano altri gruppi futuristi: quello istriano (Sambo, Boccalatte), quello di Udine (Baldini, Lami, Toffoletti, Nonino, Casoli) e soprattutto quello di Gorizia, capeggiato dalla contraddittoria personalità di Sofronio Pocarini (con lui Emilio Furlani, Emilio Casasola, i pittori Luigi Spazzapan, Veno Pilon ed Ivan Čargo).

Sia Pocarini che Carmelich furono renitenti ad un rigido inquadramento marinettiano ed attentissimi all'Europa. Non sorprende pertanto il fatto che Sanzin si autoreputasse l'unico marinettiano ortodosso e reclamasse il grado di luogotenente di Marinetti, nonché leader di quel Movimento Futurista Giuliano, che si tentò di costituire nel marzo del 1924.

Dopo un breve periodo di direzione a due, tra Pocarini e Sanzin esplosero prevedibili rivalità. La frattura venne ricomposta solo parecchi anni dopo, e dopo che Sanzin resterà solo ad assicurare la routine futuristica giuliana.

Il 1924 sarà l'anno più fertile per l'avanguardia giuliana e per la vita artistica del primo dopoguerra. Si consuma infatti in quell'anno l'esperienza culturale de "L'Aurora", rivista d'avanguardia ideata e diretta proprio da Pocarini a partire dal dicembre del

[25] Carpi, U., 1923-1925: Giornali dellavanguardismo giuliano, in Intellettuali di frontiera. Triestini a Firenze (1900-1950), cit., p. 107.

[26] „Gaudeamus Igitur", 1 gennaio 1923, cit.

[27] Carpi, U., 1923-1925: Giornali dellavanguardismo giuliano, in Intellettuali di frontiera. Triestini a Firenze (1900-1950), cit., p. 108. 
1923, con la partecipazione del primo nucleo futurista triestino, che vedeva ancora uniti Giorgio Carmelich, Emilio Mario Dolfi, Nino Jablowski e Bruno Giordano Sanzin.

Pocarini inaugura "Aurora» dedicando il primo numero a Giorgio Carmelich (che in seguito diverrà illustratore stabile della rivista), allora un adolescente locale del tutto sconosciuto e agli esordi. Infatti è proprio Pocarini ad intuire la possibilità di coordinare le giovanissime energie avanguardistiche emergenti in ambito giuliano e friulano.

[...] bisogna sottolineare anche l'intelligenza della formula adottata per "L'Aurora": materiali originali degli artisti giuliani e vasti notiziari internazionali, con limitato ricorso alle firme futuriste già prestigiose. Pocarini stabiliva così un nesso diretto provincia-Europa di stile assai giuliano e tanto più apprezzabile in una fase nella quale antiinternazionalismo ed iperitalianità costituivano le insegne sotto cui Marinetti tentava di varare in tutto il Paese, dai grandi ai piccoli centri, una sorta d'avanguardismo di regime $[\ldots]^{[28]}$.

"L’Aurora” è importante come tentativo di aggregare un tessuto avanguardistico regionale, di organizzare un gruppo di tendenza, senza però quel rigore e quella determinazione dello sperimentalismo di "Epeo". Carmelich, futurista assolutamente sui generis, imprimerà all"'Aurora" un suo stile in bilico fra Europa e provincia.

Il merito di Pocarini è stato invece quello di tentare di rompere l'isolamento del gruppo avanguardistico regionale, e di stabilire contatti di respiro nazionale e di favorire l'aggregazione "degli eccentrici e dei marginali"[29].

Negli stessi mesi veniva pubblicata a Trieste la rivista "Energie Futuriste"[30], più dimessa nella veste tipografica ma non meno interessante de "L’Aurora". I collaboratori erano gli stessi (tutti gli aderenti al movimento giuliano, ma con la nettissima prevalenza del trio di "Epeo") e vantavano rapporti internazionali, testimoniati dagli scambi con alcune tra le più importanti riviste d'avanguardia europee.

Il congresso milanese del novembre '24, con le sue celebrazioni marinettiane e il ritrovato modus vivendi col fascismo, ha fatto del futurismo un'indolore avanguardia di regime: d'ora in poi ogni conato avanguardistico si verificherà fuori del futurismo stesso, ovvero come infrazione alla sua ortodossia, ma comunque in un clima di grande difficoltà e per vie assolutamente underground. Fuori, starei per dire, dalla storia percepibile e registrabile ${ }^{[31]}$.

Carmelich e Dolfi intanto si sottraggono alle ritualità futuristiche e si orientano verso scelte costruttivistiche; Pocarini, pur non avendo mai dato gran prova di sperimentalismo rigoroso, accentua l'elemento limitativo del suo semifuturismo ed intraprende un'attività giornalistica di medio calibro.

La vicenda del gruppo giuliano e dei suoi principali animatori sta volgendo al termine, non solo perché dissolto dai destini individuali ${ }^{[32]}$, ma anche perché rimosso dall'ostilità dell'epoca e dell'ambiente.

[28] Carpi, U., 1923-1925: Giornali dellavanguardismo giuliano, in, Intellettuali di frontiera. Triestini a Firenze (1900-1950), cit., p. 112. [29] Ivi, p. 113.

[30] Su "Italia Nova", mensile triestino dell'Associazione Nazionale Tubercolotici Di Guerra, dal gennaio 1924 viene affidata a Jablowsky la responsabilità di una Rubrica Futurista, che dall'aprile prende il titolo di "Energie Futuriste". Escono con regolarità otto puntate. Ne seguiranno una nona e decima, curate però da Carmelich. La passività editoriale e il distacco di Jablowsky, porteranno Carmelich ad interrompere l'esperimento: accantonato il futurismo, le energie avanguardistiche saranno indirizzate verso altri obiettivi.

[31] Carpi, U., 1923-1925: Giornali dellavanguardismo giuliano, in, Intellettuali di frontiera. Triestini a Firenze (1900-1950), cit., p. 127.

[32] Anche per ragioni contingenti: Dolfi andrà all'università di Roma; Carmelich per via degli studi e motivi di salute peregrinerà per varie città italiane ed europee fino alla precoce morte; Jablowsky si è suicidato alla fine del 1924; Pocarini troverà la sua fine in un tragico incidente. 
Un' ostilità a tutt'oggi solo parzialmente rimossa: è singolare che, nella gran fioritura di studi e panorami sulla letteratura triestina e giuliana, non abbiano trovato alcuna attenzione non solo i pressoché segreti epeiani, ma neppure i più accessibili Pocarini e Sanzin. Dellostracismo cittadino, comunque, si lamentava "Energie Futuriste" nel numero di ottobre (p. 344), annunciando il tentativo di dar luogo ad un circuito alternativo rispetto a quello ufficiale; ma in particolare protestava il Dolfi (Nuove vie delle arti plastiche, in "L'Aurora", marzo 1924), che denunciava il fronte comune di "parassitismo dei buoni a nulla", di "critici reazionari" e di "quella gran bestia che è il pubblico" ${ }^{[33]}$.

Con il concludersi del breve ciclo liceale di Dolfi e Carmelich, lofficina avanguardistica inaugurata da "Epeo" declina verso la chiusura, ma non senza un estremo sprazzo.

Nel 1925 esce, come unepigrafe, "25" di cui, stando agli attuali esiti di ricerca, pare sia stato pubblicato solo il primo numero del gennaio 1925, in cui ritroviamo insieme Carmelich e Dolfi che dedicano l'edizione al cartellonismo ovvero all'arte della réclame.

In campo letterario intanto a Trieste avevano pubblicato Vladimiro Miletti, Aristide Mattiussi, Trisno (Tristano Pantaloni), Marcello Clari, Ugo Carà, Matia Lupieri, Ferruccio Demanis, Wanda Wulz e altri, meno noti quali futuristi, ma di certo influenzati dal movimento di Marinetti.

Dopo la morte improvvisa di Pocarini (1934), Crali assunse a Gorizia l'impegno di organizzatore culturale e rappresentante del Futurismo, mentre a Trieste proseguì con continuità l'opera di Sanzin. Entrambi mantennero stretti rapporti, fino alla fine (occorsa nel 1944) con il capo storico e fondatore del Futurismo, Filippo Tommaso Marinetti.

\section{BIBLIOGRAFIA}

AA.VV., 1914, I manifesti del Futurismo, Edizioni Lacerba, Firenze.

ARMELlINI, G., COLOMBO, A., 1999, Guida alla letteratura italiana, Zanichelli, Bologna.

ARMELliNI, G., COLOMBO, A., 2005, Letteratura. Letterature. Antologia Primo Novecento, Vol. G, Zanichelli, Bologna.

CARPI, U., 1985, Personaggi e vicende della letteratura giuliana d'avanguardia negli anni Venti, in Frontiere d'avanguardia. Gli anni del Futurismo nella Venezia Giulia, Catalogo della mostra a cura di Bruno Passamani e Umberto Carpi, Musei Provinciali, febbraio-aprile, Gorizia.

CARPI, U., 1985, 1923-1925: Giornali dell'avanguardismo giuliano, in PERTICI, R. (a cura di), Intellettuali di frontiera. Triestini a Firenze (1900-1950), Atti del Convegno (18-20 marzo 1983), Vol. I, Olschki, Firenze, 97-128.

DE MARIA, L., DONDI, L., 1994, (a cura di), Marinetti e i Futuristi, Garzanti, Milano.

FANTASIA, R., TALLINI, G., 2004, Poesia e rivoluzione. Simbolismo, Crepuscolarismo, Futurismo, Franco Angeli, Milano.

Gaudeamus Igitur, n. 2, Trieste, 9 dicembre 1922.

[33] Carpi, U., 1923-1925: Giornali dellavanguardismo giuliano, in Intellettuali di frontiera. Triestini a Firenze (1900-1950), cit., p. 128, nota 50 . 
GENTILE, E., 2000, Political Futurism and the Myth of the Italian Revolution, in BERGHAUS, G. (a cura di), European cultures. Studies in literature and the Arts, International Futurism in arts and literature, de Gruyter, Berlin-New York.

GIBELLINI, P., OLIVA, G., TESIO, G., 1990, Lo spazio letterario. Storia e geografia della letteratura italiana, La Scuola, Brescia.

GUGLIELMINO, S., 1990, (4). Guida al Novecento. Profilo letterario e antologia, Principato, Milano.

HACE, CITRA, R., 1996, Carolus L. Cergoly Serini dietro le quinte della pagina, Istituto Italiano di Cultura - Zagreb, EDIT, Fiume-Rijeka.

MARINETTI, F. T., 1968, Teoria e invenzione futurista, (a cura di) DE MARIA, L., Mondadori, Milano.

PASSAMANI, B., 1985, Dall'alcova d’acciaio al Tank ai Macchi 202. Energie futuriste e costruttiviste tra rivolta, utopia e realtà alla frontiera giulia, in PASSAMANI, B., CARPI, U., (a cura di), Frontiere d’avanguardia. Gli anni del Futurismo nella Venezia Giulia, Catalogo della mostra Musei Provinciali, febbraio-aprile, Gorizia. P. 18.

RICOV, J., 2004, Talijanski futurizam s predcima i potomstvom, Hrvatsko književno društvo sv. Jeronima, Zagabria-Zagreb.

ROJIĆ, S., 2011, Lombra del Futurismo nei Balcani doggi, in FRASSICA, P. (a cura di), Shades of Futurism. Futurismo in ombra, Atti del Convegno internazionale Princeton, 9-10 ottobre 2009, Interlinea, Novara, p. 168.

SALARIS, C., 1985, Storia del futurismo, Editori riuniti, Roma.

SANZIN, B. G., 1975, Tappe autobiografiche essenziali, in ES, n. 3, febbraiomaggio, 59-71.

VIOLA, G. E., 1990, Gli anni del Futurismo. La poesia italiana nelletà delle avanguardie, Studium, Roma.

\section{FUTURIZAM U JULIJSKOJ KRAJINI}

Marinettijev futurizam, zasigurno, predstavlja jedan od najagresivnijih književnih, umjetničkih i političkih talijanskih pokreta dvadesetoga stoljeća. Nastojeći prekinuti sa svim tradicijama i zadanim vrijednostima kroz tehnicizam, brzinu i borbu proklamirao je najtješnju privrženost aktivizmu i dinamici suvremenoga svijeta.

U novoj restauracijskoj atmosferi talijanske književnosti u dvadesetim godinama prošloga stoljeća područja periferije, između kojih je i Trst, doživjele su neku vrstu kasnoga avangardizma, a dio julijanske književne produkcije doživjet će i doba kasnoga futurizma.

Julijanski futurizam predstavlja se artikulirano kroz specifičnost, takozvane „pogranične" te srednjoeuropske kulture, kao i kroz prisutnost talijansko-slovenske antropološke i kulturne komponente, koje karakteriziraju ovu regiju.

Ključne riječi: futurizam, Italija, pokret, Julijska krajina 


\section{FUTURISM IN JULIAN MARCH}

Marinetti's futurism is certainly one of the most aggressive literary, artistic and political Italian movements in the $20^{\text {th }}$ century. Trying to abandon all traditions and values though technicism, speed and fight, it proclaimed the strongest attachment to activism and dynamics in the contemporary world.

In the new restoration atmosphere of Italian literature in the 1920s, the peripheral areas, with Trieste being one of them, experienced some sort of late avant-gardism, while a part of the Julian literary production even reached late futurism.

The Julian futurism is articulated through specific features of the so-called 'border-line' as well as Middle European culture, also through the presence of ItalianSlovene anthropological and cultural component, so characteristic for this region.

Key words: Futurism, Italy, movement, Julian March (Venezia-Giulia) 